\title{
Birth characteristics and childhood carcinomas
}

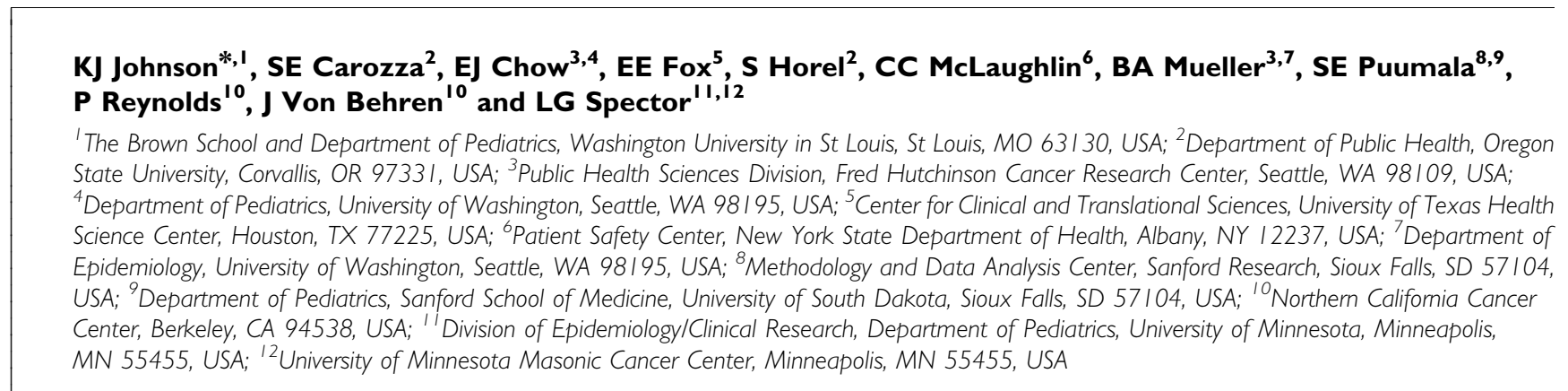

BACKGROUND: Carcinomas in children are rare and have not been well studied.

METHODS: We conducted a population-based case-control study and examined associations between birth characteristics and childhood carcinomas diagnosed from 28 days to 14 years during 1980-2004 using pooled data from five states (NY, WA, MN, $\mathrm{TX}$, and CA) that linked their birth and cancer registries. The pooled data set contained 57966 controls and 475 carcinoma cases, including 159 thyroid and 126 malignant melanoma cases. We used unconditional logistic regression to calculate odds ratios (ORs) and $95 \%$ confidence intervals (Cls).

RESULTS: White compared with 'other' race was positively associated with melanoma ( $\mathrm{OR}=3.22,95 \% \mathrm{Cl}$ I.33-8.33). Older maternal

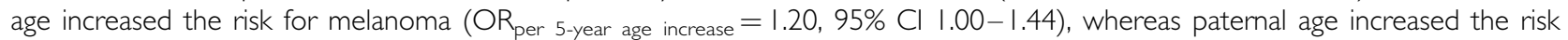

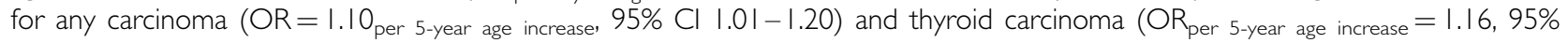
$\mathrm{Cl} 1.01-1.33)$. Gestational age $<37$ vs $37-42$ weeks increased the risk for thyroid carcinoma $(\mathrm{OR}=1.87,95 \% \mathrm{Cl} 1.07-3.27)$. Plurality, birth weight, and birth order were not significantly associated with childhood carcinomas.

CONCLUSION: This exploratory study indicates that some birth characteristics including older parental age and low gestational age may be related to childhood carcinoma aetiology.

British Journal of Cancer (201 I) 1 05, 1396- I40 I. doi:I0.1038/bjc.20 I I.359 www.bjcancer.com

Published online 13 September 201।

(C) 201 I Cancer Research UK

Keywords: paediatric; carcinoma; melanoma; thyroid; risk

In contrast to adults, carcinomas in children are extremely rare with an estimated annual incidence among those $0-14$ years of age of 6.3 cases per million (Surveillance, Epidemiology, and End Results, 2008). The incidence of childhood carcinomas increases with age with most cases being diagnosed after age 14 years. The major childhood carcinoma subtypes are renal, hepatic, gonadal, adrenocortical, thyroid, nasopharyngeal, and malignant melanoma (Steliarova-Foucher et al, 2005). Thyroid carcinoma and melanoma comprise $60 \%$ of cases (Surveillance, Epidemiology, and End Results, 2008). As is the case for adults, there is a female predominance for both thyroid carcinoma and malignant melanoma (Pizzo and Poplack, 2006). Both of these subtypes are more common in whites than blacks. The incidence of paediatric malignant melanoma has increased over recent decades for unknown reasons. Notably, from 1992 to 2007, the incidence of paediatric malignant melanoma has increased significantly at an average rate of $4.8 \%$ per year (Surveillance, Epidemiology, and End Results, 2008).

*Correspondence: Dr KJ Johnson;

E-mail: kijohnson@brownschool.wustl.edu

Received I8 April 20 I ; revised 22 July 20 II; accepted I4 August 20 I ; published online 13 September 201 I
The current understanding of the aetiology of the two most common paediatric carcinomas, thyroid carcinoma and malignant melanoma, is predominantly based on case-series reports and studies of rare syndromes. Syndromes associated with thyroid carcinomas include the following: familial adenomatous polyposis, multiple endocrine neoplasia type 2, Cowden syndrome, Carney complex, Pendred syndrome, and Werner syndrome (Richards, 2010). Xeroderma pigmentosum, a DNA repair disorder, is associated with an increased risk of paediatric melanoma (Pizzo and Poplack, 2006). Germline mutations in CDKN2A and CDK4 (Udayakumar and Tsao, 2009) have been linked to familial melanoma, however mutations in both of these genes have been infrequently reported in young onset melanoma (Tsao et al, 2000; Berg et al, 2004; Debniak et al, 2008). Other biological risk factors for childhood melanoma include giant congenital melanocytic nevi, familial dysplastic nevus syndrome, fair skin, light hair colour, blue or green eyes, immunosuppression, and atypical or numerous nevi (Jen et al, 2009). Ionising radiation exposure is an established risk factor for adult and childhood thyroid carcinoma (Ron, 2007). Numerous adult melanoma studies have noted an increased risk in relation to sun exposure (Schottenfeld and Fraumeni, 2006), however the extent to which this is associated with childhood melanoma is unclear (Whiteman et al, 1997; Youl et al, 2011). 
Early life is thought to be critical for cancer development in children (Boice and Miller, 1999; Greaves, 2003). To our knowledge, no studies have specifically examined the association between birth characteristics and childhood carcinomas. Using data from a registry-based linkage study, our objective was to conduct an exploratory analysis of associations between factors recorded in birth records and childhood carcinomas, both overall and separately for the two major subtypes (thyroid carcinoma and malignant melanoma). Because of the size and scope of this study, it may offer additional clues to the aetiology of carcinomas in children.

\section{MATERIALS AND METHODS}

Study procedures have been reported in detail previously (Johnson et al, 2009; Puumala et al, 2009; Spector et al, 2009; Carozza et al, 2010; Chow et al, 2010). Institutional Review Board approvals were obtained from each state's health department and participating institutions before conducting the study. Individuals who were diagnosed with childhood cancer during 1980-2004 were identified through the population-based central cancer registries of California, Minnesota, New York (excluding New York City), Texas, and Washington states. Briefly, childhood cancer cases in each state's cancer registry were linked to their respective birth certificates using sequential deterministic or probabilistic record linkage (Jaro, 1995). Cases were classified according to the International Classification of Childhood Cancer 3rd edition (Steliarova-Foucher et al, 2005). All cases of melanoma were malignant. Age at diagnosis in months was obtained from cancer registry records. Controls were randomly selected from each state's birth registry in ratios to cases that varied from 1 to 10 . Frequency matching was used in four states and individual matching in one (CA); year of birth was a matching factor in all states although two also matched on sex (CA, TX).

Additional criteria were applied before pooling to ensure uniformity of data. Cases that were also selected as controls in the case-cohort sampling strategy employed by Minnesota and New York were excluded from the control group in this analysis. California cases included those diagnosed at $<28$ days old, whom we subsequently excluded for consistency with other states. Subjects with reported Down syndrome $(n=100)$ were excluded, although it should be noted that Down syndrome was not recorded in Texas before 1984 or in Washington before 1989. The final pooled data set included 17672 cases and 57966 controls.

\section{Statistical analyses}

Odds ratios (ORs) and 95\% confidence intervals (CIs) were calculated by unconditional logistic regression (SAS version 9.1, SAS, Cary, NC, USA); individual matching in the California data set was broken for this reason. Race was categorised as white and non-white using maternal race as a proxy for the child's race. We modelled associations with parental age both as continuous variables in 5 -year increments and as categorical variables $(<25$, $25-29,30-34$, and $\geqslant 35$ years). Categorical variables were used to assess the appropriateness of the log linear model for associations between parental age and childhood carcinomas. Each parent's age was examined in separate models and in models that included maternal and paternal age variables. Gestational age was categorised as preterm ( $<37$ weeks), term ( $37-42$ weeks), and post-term ( $>42$ weeks). Birth weight was modelled as a continuous variable in 500-g increments and in categories using the following cutoffs for low $(<2500 \mathrm{~g})$, normal $(2500-4000 \mathrm{~g})$, and high birth weight $(>4000 \mathrm{~g}$ ). All models were adjusted for state and the matching variables sex and birth year. Because knowledge about risk factors for childhood carcinomas is sparse we included all available potential confounders of associations between birth characteristic and childhood carcinoma. Therefore, in addition to state, birth year, and sex, models included gestational age, plurality, birth weight, birth order and maternal or paternal age. Effect modification on the multiplicative scale was assessed by including the cross product of the variables of interest in the logistic regression model. We included only subjects with complete data on all variables. The percentage of cases and controls with missing data on the variables included in this analysis was similar for sex ( $0 \% v s$. $0.02 \%)$, maternal age $(0 \% v s$ $0.04 \%)$, paternal age ( $11 \%$ vs $11 \%)$, plurality ( $0 \%$ vs $0.6 \%)$, birth weight $(0 \%$ vs $0.6 \%)$, gestational age $(6.1 \%$ vs $4.6 \%)$, birth order (3.4\% vs $2.6 \%)$, and maternal race (3.2\% vs $1.5 \%)$. The number of subjects that were excluded from each model is detailed in the table 2 footnote. ORs were considered statistically significant if the $P$-value was $\leqslant 0.05$.

\section{RESULTS}

A total of 475 children diagnosed with carcinomas between 1980 and 2004 were identified in the pooled data set (Table 1). Thyroid carcinomas $(n=159)$ were most common followed by melanomas $(n=126)$, and together constituted more than half $(60 \%)$ of the carcinomas in this study. The median diagnosis age was 11.4 years for carcinomas overall (data not shown) with most cases being diagnosed between 10 and 14 years overall and for most subtypes. More females were diagnosed with carcinomas than males particularly for thyroid carcinomas. By race, there were notable differences for melanoma where very few cases were non-white. This is in contrast to renal cell carcinomas and nasopharyngeal carcinomas where a larger percentage were non-white, $44 \%$ and $38 \%$, respectively, compared with controls $(14.7 \%$ (data not shown)). Because of the small number of cases for most cancer sites, associations with birth characteristics are presented for all carcinomas combined and for the two sites with 100 or more cases (malignant melanoma and thyroid carcinoma).

Maternal white race compared with 'other' race conferred a significantly increased risk for melanoma (OR $=3.27,95 \%$ CI $1.33-8.06)$ but not for all carcinomas or thyroid carcinoma (Table 2).

Maternal age modelled as a continuous variable was associated with a marginally significant increased risk for childhood carcinomas overall $\left(\mathrm{OR}_{\text {per } 5 \text {-year increase }}=1.09,95 \%\right.$ CI $\left.0.99-1.20\right)$. Both thyroid carcinoma and melanoma showed a positive linear maternal age effect that was significant for melanoma $\left(\mathrm{OR}_{\text {per } 5 \text {-year }}\right.$ increase $=1.20,95 \%$ CI $1.00-1.44)$. In models that categorised maternal age, there was a consistent nonsignificant pattern of increasing risk with advancing maternal age category for all carcinomas. However, there was some evidence for an association of older maternal age for malignant melanoma where all maternal age categories were associated with elevated risks compared with the reference group and for thyroid carcinoma in the offspring of women born to mothers $\geqslant 35 v s<25$ years $(\mathrm{OR}=1.87,95 \%$ CI $1.01-3.48)$. There was no significant effect modification by birth year category (1975-1984 vs 1985-2004) between maternal age modelled as a continuous variable and all carcinomas, thyroid carcinomas, or malignant melanomas.

Paternal age modelled as a continuous variable was associated with a significant positive linear trend for childhood carcinomas overall $\left(\mathrm{OR}_{\text {per } 5 \text {-year increase }}=1.10,95 \% \mathrm{CI} 1.01-1.20\right)$ and thyroid carcinoma $\left(\mathrm{OR}_{\text {per }} 5\right.$-year increase $\left.=1.16,95 \% \mathrm{CI} 1.01-1.33\right)$ and a nonsignificant positive linear trend for malignant melanoma $(\mathrm{OR}=1.13,95 \%$ CI $0.96-1.32)$. In models where paternal age was modelled categorically, the risk for all childhood carcinomas and thyroid carcinomas specifically generally increased with increasing paternal age, consistent with the linear model of paternal age; however all estimates were imprecise. There was no significant effect modification by birth year category (1975-1984 
Table I Characteristics of childhood carcinoma cases in the pooled data set

\begin{tabular}{|c|c|c|c|c|c|c|c|c|c|c|}
\hline & $\begin{array}{c}\text { Total } \\
(n=475)\end{array}$ & $\begin{array}{l}\text { Thyroid } \\
(n=159)\end{array}$ & $\begin{array}{c}\text { Malignant } \\
\text { melanoma } \\
(n=126)\end{array}$ & $\begin{array}{l}\text { Hepato- } \\
\text { cellular } \\
(n=4 I)\end{array}$ & $\begin{array}{l}\text { Adreno- } \\
\text { cortical } \\
(n=21)\end{array}$ & $\begin{array}{c}\text { Renal } \\
\text { cell } \\
(n=16)\end{array}$ & $\begin{array}{c}\text { Naso- } \\
\text { pharyngeal } \\
(n=13)\end{array}$ & $\begin{array}{c}\text { Gonadal } \\
(n=9)\end{array}$ & $\begin{array}{c}\text { Non-melanoma } \\
\text { skin } \\
(n=5)\end{array}$ & $\begin{array}{l}\text { Others and } \\
\text { unspecified } \\
(n=85)^{\mathrm{a}}\end{array}$ \\
\hline $5-9$ & 20.6 & 20.1 & 17.5 & 24.4 & 23.8 & 37.5 & 23.1 & 11.1 & 60.0 & 18.8 \\
\hline $10-14$ & 61.7 & 75.5 & 55.6 & 34.2 & 9.5 & 62.5 & 76.9 & 77.8 & 40.0 & 68.2 \\
\hline
\end{tabular}

${ }^{\mathrm{a}} 91 \%(n=77)$ were found in other tissue sites and $9 \%(n=8)$ were classified as having an unknown primary site. ${ }^{\mathrm{b}} \mathrm{A}$ total of 15 cases $(6$ thyroid carcinomas, 5 malignant melanomas, I hepatocellular carcinoma, 2 nasopharyngeal carcinomas, and I gonadal carcinoma) had missing data on maternal race.

Table 2 Associations between birth characteristics and any childhood carcinoma, thyroid carcinomas, and malignant melanomas diagnosed from $0-14$ years

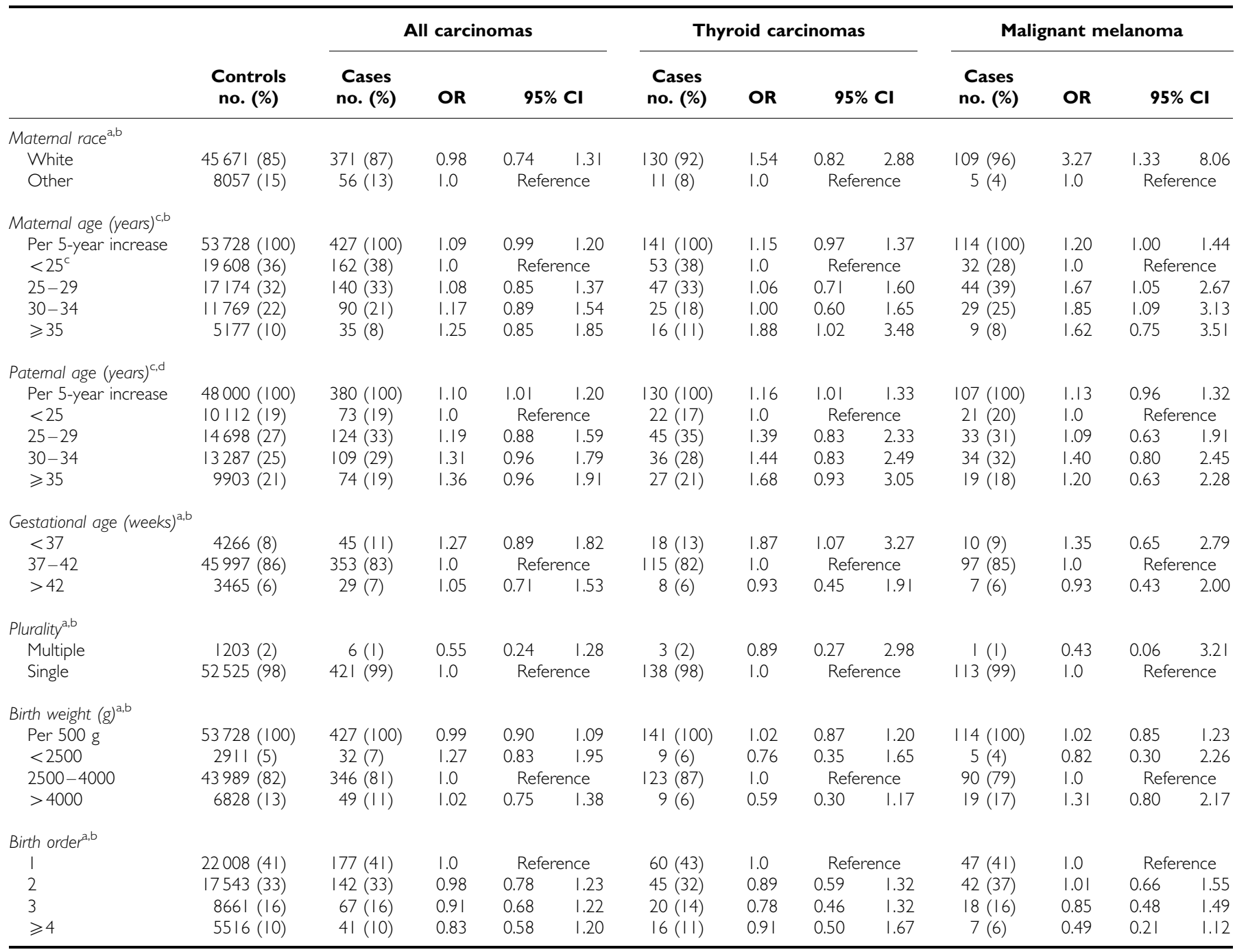

Abbreviations: $\mathrm{Cl}=$ confidence interval; OR = odds ratio. ${ }^{a}$ Adjusted for state (CA, WA, MN, NY, TX), birth year category (1970-85, 1986-89, 1990-93, 1994-2004), materna race (white, other), sex (male, female), maternal age as a continuous variable, gestational age ( $<37,37-42,>42$ weeks), plurality (multiple, single), birth weight $(<2500,2500-4000$, $>4000 \mathrm{~g})$, and birth order $(\mathrm{I}, 2,3, \geqslant 4)$. 4238 controls and 48 total carcinoma cases ( $n=18$ thyroid carcinoma and 12 malignant melanoma cases) were excluded due to missing data on one or more covariates. 'Adjusted for state (CA, WA, MN, NY, TX), birth year category (1970-85, 1986-89, 1990-93, 1994-2004), maternal race (white, other), sex (male, female), gestational age (37-42, <37, >42 weeks), plurality (multiple, single), birth weight ( $<2500,2500-4000,>4000 \mathrm{~g}$ ), and birth order ( $1,2,3, \geqslant 4$ ). ${ }^{d} 9966$ controls and 95 total carcinoma cases (29 thyroid carcinoma and 19 malignant melanoma cases) were excluded due to missing data on one or more covariates.

vs 1985-2004) between paternal age modelled as a continuous variable and all carcinomas, thyroid carcinomas, or malignant melanomas. In models that included maternal and paternal age, the independent effects of each were attenuated (data not shown).
Plurality, birth weight, and birth order were not significantly associated with all carcinomas, thyroid carcinoma, or melanoma. Gestational age $<37$ weeks vs $37-42$ weeks was associated with a significant increased risk of thyroid carcinoma $(\mathrm{OR}=1.87,95 \%$ 
CI 1.07-3.27). Also of note is that the risk for melanoma appeared to decrease in relation to increasing birth order category, albeit CIs were wide; for birth orders of four or higher $v s$ first born the OR was 0.49 (95\% CI $0.21-1.12)$.

We also explored whether associations between parental age and childhood carcinomas varied by age of diagnosis and sex (Supplementary Table 1). The risk estimates for the association between parental age and childhood carcinoma for those diagnosed $\leqslant 11$ years of age $v s>11$ years of age were generally similar. However, we note that there was a consistently stronger parental age association for individuals in the younger $v s$ older age category. Associations between parental age and carcinomas between sexes were not statistically significantly different.

\section{DISCUSSION}

In addition to the previously documented risk factor for melanoma of white race, (Schottenfeld and Fraumeni, 2006) we observed a weak parental age effect for both thyroid carcinomas and melanomas. For thyroid carcinomas, the data suggest a paternal age effect, whereas for melanomas the data support a maternal age effect. We also observed an increased risk of thyroid carcinoma in association with gestational age $<37$ weeks and a decreased risk of melanoma with increasing birth order.

Older parental age has been associated with a modest risk increase for several of the more common childhood cancers including leukaemia, central nervous system tumours, and Wilm's tumour in some but not all of the larger studies (Dockerty et al, 2001; Yip et al, 2006; Johnson et al, 2009; Schuz et al, 2011). For malignant melanoma diagnosed at any age, no increased risk was found in association with maternal or paternal age in a study of all Norwegian cases $(n=709)$ born between 1967 and 1986 followed until 31 December 2003 (Franco-Lie et al, 2008). A significant positive trend with increasing maternal age at first birth but not birth of the index child was found in a study of 2594783 Danes that included 1674 incident malignant melanoma cases followed from 1968 to 2002 who were born between 1950 and 2002 (Olesen et al, 2009). Although it involved older cases than those in our study, a Swedish study reported a significant $30 \%$ excess risk of malignant melanoma in the offspring (aged 15-53 years) of mothers who were $>40$ years compared with those $<20$ years at their time of birth, whereas a $28 \%$ decreased risk was reported in the offspring of fathers who were $>40 v s<25$ at the time of their birth. No significant associations were found between maternal or paternal age and thyroid cancer (Hemminki and Kyyronen, 1999), while another study also found a protective effect of paternal age on thyroid carcinoma risk (Galanti et al, 1997). The vast majority of individuals in these studies were $>15$ years at the time of their cancer diagnosis.

Several hypotheses have been proposed to explain the increased risks of various diseases in offspring in relation to advanced parental age. The most plausible explanation for a paternal age effect is the accumulation of de novo mutations that are thought to occur in aging gametes that divide throughout the male lifetime (Crow, 2000). It is possible that a paternal age effect for young onset carcinomas could be mediated by de novo mutations in the paternal germline in genomic regions that predispose to carcinomas. The accumulation of mutations as an underlying explanation for a maternal age effect is less likely because oocyte cell division is completed before birth. A maternal age effect is well known for Down syndrome, which is most commonly caused by three copies of chromosome 21, with the extra copy usually of maternal origin (Crow, 2000). However, we are not aware of any evidence supporting an excess of germline chromosomal abnormalities in childhood carcinoma cases. Other plausible biological hypotheses for a maternal age effect on disease risk in the offspring could involve epigenetic and mitochondrial mechanisms. For example, differences in gene expression have been noted between young and old oocytes (Hamatani et al, 2004; Steuerwald et al, 2007; Pan et al, 2008). The extent to which these differences are transmitted to offspring and could explain an increased childhood cancer risk of any subtype including carcinomas has not been well studied. However, transgenerational inheritance of epimutations is plausible with the report of a maternally inherited hypermethylated $\mathrm{MLH1}$ allele in a subject with early-onset colorectal carcinoma (Hitchins et al, 2007). With regard to mitochondrial mechanisms, it is plausible that maternally transmitted mitochondrial DNA mutations that increase with age could be linked to cancer in the offspring (Eichenlaub-Ritter et al, 2011).

Although based on small numbers, our data suggested stronger associations between older parental age and childhood carcinomas in younger individuals which would be consistent with the hypothesis of an underlying genetic predisposition caused by an increased frequency of germline mutations in older parents (Arver et al, 2000). However, it is also possible that advanced parental age could be a proxy for other non-genetic factors that are related to childhood cancer risk. Because our data set contained only a limited number of birth record variables, we could not assess whether the parental age associations were confounded by factors plausibly related to older parental age and the carcinomas under study such as vacation sun exposure for melanoma (Jen et al, 2009).

We observed a nearly two-fold increased risk of thyroid carcinoma in individuals born $<37$ weeks. To our knowledge, no previous study has examined the association between thyroid carcinoma and gestational age. Further study is necessary to determine if this finding could be the related to medical exposures that occur more frequently in pre-term infants, such as diagnostic radiation (Pizzo and Poplack, 2006; Lai and Bearer, 2008; Papadopoulou and Efthimiou, 2009).

The association between birth order and malignant melanoma has been examined in two studies conducted in Denmark and Sweden (Hemminki and Mutanen, 2001; Olesen et al, 2009). In $\sim 2.5$ million Danes born from 1950 to 2002 that were followed for incidence cutaneous malignant melanoma diagnoses $(n=1674)$ at all ages (i.e., children and adults), individuals with birth orders of five or higher had a significantly reduced risk of melanoma (IRR $=0.24,95 \%$ CI $0.06-1.01$ ); however this result was based on only two cases in the highest birth order category (Olesen et al, 2009). In Sweden, in 1.38 million individuals $<55$ years who were born during 1958-1996 and followed for melanoma diagnoses $(n=1101)$, a nonsignificant reduction in risk of $5 \%$ was observed per one unit increase in birth order (Hemminki and Mutanen, 2001). Further study is necessary to evaluate the role of birth order in malignant melanoma.

A strength of our study is that it is large and population based with birth characteristic data obtained before diagnosis. To our knowledge, this is the first study to specifically address the relationship between birth characteristics and paediatric carcinomas. Our study also has limitations. Although the accuracy of some characteristics may not be optimal, the variables that we used generally have high validity (Northam and Knapp, 2006) and any misclassification should be non-differential with regard to case $v s$ control status. Because childhood carcinomas are rare, our statistical power to detect weak or modest associations was limited and thus our results should be considered exploratory. We also did not have follow-up of our control population, which could have resulted in missed cases. For example, if a control moved out of state and developed a childhood carcinoma, he/she would have not been included as a case in our data set. However, because childhood carcinomas are very rare it is unlikely that any controls developed this type of cancer; therefore it is also unlikely that this type of bias affected our results. Missing data could have biased the results if those with complete data 
were systematically different from those with missing data. The percentage of cases and controls with missing data on the variables included in this analysis was similar and generally $<10 \%$ (with the exception of paternal age at $11 \%$ ) making it seem unlikely that missing data had a strong influence on our results. Finally, it is possible that chance variation could be an explanation for our results.

In summary, we found that our results for childhood carcinomas reflected the same patterns seen for adults of a positive association between white race and melanoma. We found no evidence for an association between most other birth characteristics and childhood carcinomas with the possible exception of a relationship between birth order and melanoma, low gestational age and thyroid carcinoma, and a parental age effect for childhood carcinomas overall, and melanoma and thyroid carcinoma specifically.

\section{ACKNOWLEDGEMENTS}

We wish to acknowledge the programming assistance of Eric Elkin, Susan Hurley, John Soler, and Bill O'Brien. We also would like to thank the Washington State Department of Health, the Minnesota Cancer Surveillance System, and other collaborating institutions for allowing data access. This study was funded by Children's Cancer Research Fund, Minneapolis, MN; National Cancer Institute (N01$\mathrm{CN}-05230$ to WA, R01CA71745 to CA, R01CA92670 to TX, T32 CA099936 to MN); Fred Hutchinson Cancer Research Center; Centers for Disease Control and Prevention's National Program of Cancer Registries by cooperative agreement (U58DP000783-01 to NY).

Supplementary Information accompanies the paper on British Journal of Cancer website (http://www.nature.com/bjc)

\section{REFERENCES}

Arver B, Du Q, Chen J, Luo L, Lindblom A (2000) Hereditary breast cancer: a review. Semin Cancer Biol 10(4): 271-288

Berg P, Wennberg AM, Tuominen R, Sander B, Rozell BL, Platz A, Hansson $\mathrm{J}$ (2004) Germline CDKN2A mutations are rare in child and adolescent cutaneous melanoma. Melanoma Res 14(4): 251-255

Boice Jr JD, Miller RW (1999) Childhood and adult cancer after intrauterine exposure to ionizing radiation. Teratology 59(4): 227-233

Carozza SE, Puumala SE, Chow EJ, Fox EE, Horel S, Johnson KJ, McLaughlin CC, Reynolds P, Von Behren J, Mueller BA Spector LG (2010) Parental educational attainment as an indicator of socioeconomic status and risk of childhood cancers. Br J Cancer 103(1): $136-142$

Chow EJ, Puumala SE, Mueller BA, Carozza SE, Fox EE, Horel S, Johnson KJ, McLaughlin CC, Reynolds P, Von Behren J, Spector LG (2010) Childhood cancer in relation to parental race and ethnicity: a 5-state pooled analysis. Cancer 116(12): 3045-3053

Crow JF (2000) The origins, patterns and implications of human spontaneous mutation. Nat Rev Genet 1(1): 40-47

Debniak T, van de Wetering T, Scott R, Nagay L, Cybulski C, Gorski B, Jakubowska A, Gronwald J, Masojc B, Huzarski T, Byrski T, Nej-Wolosiak K, Kladny J, Maleszka R, Lubinski J (2008) Low prevalence of CDKN2A/ARF mutations among early-onset cancers of breast, pancreas and malignant melanoma in Poland. Eur J Cancer Prev 17(5): $389-391$

Dockerty JD, Draper G, Vincent T, Rowan SD, Bunch KJ (2001) Case-control study of parental age, parity and socioeconomic level in relation to childhood cancers. Int J Epidemiol 30(6): 1428-1437

Eichenlaub-Ritter U, Wieczorek M, Luke S, Seidel T (2011) Age related changes in mitochondrial function and new approaches to study redox regulation in mammalian oocytes in response to age or maturation conditions. Mitochondrion 11(5): 783-796

Franco-Lie I, Iversen T, Robsahm TE, Abdelnoor M (2008) Birth weight and melanoma risk: a population-based case-control study. Br J Cancer 98(1): $179-182$

Galanti MR, Ekbom A, Grimelius L, Yuen J (1997) Parental cancer and risk of papillary and follicular thyroid carcinoma. Br J Cancer 75(3): 451 - 456

Greaves M (2003) Pre-natal origins of childhood leukemia. Rev Clin Exp Hematol 7(3): 233-245

Hamatani T, Falco G, Carter MG, Akutsu H, Stagg CA, Sharov AA, Dudekula DB, VanBuren V, Ko MS (2004) Age-associated alteration of gene expression patterns in mouse oocytes. Hum Mol Genet 13(19): $2263-2278$

Hemminki K, Kyyronen P (1999) Parental age and risk of sporadic and familial cancer in offspring: implications for germ cell mutagenesis. Epidemiology 10(6): 747-751

Hemminki K, Mutanen P (2001) Birth order, family size, and the risk of cancer in young and middle-aged adults. Br J Cancer 84(11): 1466-1471

Hitchins MP, Wong JJ, Suthers G, Suter CM, Martin DI, Hawkins NJ, Ward RL (2007) Inheritance of a cancer-associated MLH1 germ-line epimutation. N Engl J Med 356(7): 697-705

Jaro MA (1995) Probabilistic linkage of large public health data files. Stat Med 14(5-7): $491-498$
Jen M, Murphy M, Grant-Kels JM (2009) Childhood melanoma. Clin Dermatol 27(6): 529-536

Johnson KJ, Carozza SE, Chow EJ, Fox EE, Horel S, McLaughlin CC, Mueller BA, Puumala SE, Reynolds P, Von Behren J, Spector LG (2009) Parental age and risk of childhood cancer: a pooled analysis. Epidemiology 20(4): $475-483$

Lai TT, Bearer CF (2008) Iatrogenic environmental hazards in the neonatal intensive care unit. Clin Perinatol 35(1): 163-181, ix

Northam S, Knapp TR (2006) The reliability and validity of birth certificates. J Obstet Gynecol Neonatal Nurs 35(1): 3-12

Olesen AV, Parner ET, Mortensen PB, Sorensen HT, Olsen J (2009) Prenatal risk factors for cutaneous malignant melanoma: follow-up of 2594783 Danes born from 1950 to 2002. Cancer Epidemiol Biomarkers Prev 18(1): $155-161$

Pan H, Ma P, Zhu W, Schultz RM (2008) Age-associated increase in aneuploidy and changes in gene expression in mouse eggs. Dev Biol 316(2): $397-407$

Papadopoulou F, Efthimiou E (2009) Thyroid cancer after external or internal ionizing irradiation. Hell J Nucl Med 12(3): 266-270

Pizzo PA, Poplack DG (2006) Principles and practice of pediatric oncology, 5th edn. Lippincott Williams \& Wilkins: Philadelphia

Puumala SE, Carozza SE, Chow EJ, Fox EE, Horel S, Johnson KJ, McLaughlin C, Mueller BA, Reynolds P, Von Behren J, Spector LG (2009) Childhood cancer among twins and higher order multiples. Cancer Epidemiol Biomarkers Prev 18(1): 162-168

Richards ML (2010) Familial syndromes associated with thyroid cancer in the era of personalized medicine. Thyroid 20(7): 707-713

Ron E (2007) Thyroid cancer incidence among people living in areas contaminated by radiation from the Chernobyl accident. Health Phys 93(5): $502-511$

Schottenfeld D, Fraumeni JF (2006) Cancer epidemiology and prevention, 3rd edn. Oxford University Press: Oxford, New York

Schuz J, Schmidt LS, Kogner P, Lahteenmaki PM, Pal N, Stokland T, Schmiegelow K (2011) Birth characteristics and Wilms tumors in children in the Nordic countries: a register-based case-control study. Int J Cancer 128(9): 2166-2173

Surveillance, Epidemiology, and End Results (SEER) Program (Internet). SEER ${ }^{*}$ Stat Database: Incidence - SEER 13 Regs Limited-Use: Bethesda (MD), Nov 2008 Sub (1992-2007) <Katrina/Rita Population Adjustment $>$ - Linked To County Attributes - Total U.S., 1969-2006 Counties, National Cancer Institute, DCCPS, Surveillance Research Program, Cancer Statistics Branch, released April 2009, based on the November 2009 submission. Available from: http://www.seer.cancer.gov

Spector LG, Puumala SE, Carozza SE, Chow EJ, Fox EE, Horel S, Johnson KJ, McLaughlin CC, Reynolds P, Behren JV, Mueller BA (2009) Cancer risk among children with very low birth weights. Pediatrics 124(1): $96-104$

Steliarova-Foucher E, Stiller C, Lacour B, Kaatsch P (2005) International classification of childhood cancer, third edition. Cancer 103(7): $1457-1467$

Steuerwald NM, Bermudez MG, Wells D, Munne S, Cohen J (2007) Maternal age-related differential global expression profiles observed in human oocytes. Reprod Biomed Online 14(6): 700-708 
Tsao H, Zhang X, Kwitkiwski K, Finkelstein DM, Sober AJ, Haluska FG (2000) Low prevalence of germline CDKN2A and CDK4 mutations in patients with early-onset melanoma. Arch Dermatol 136(9): 1118-1122

Udayakumar D, Tsao H (2009) Melanoma genetics: an update on riskassociated genes. Hematol Oncol Clin North Am 23(3): 415-429, vii

Whiteman DC, Valery P, McWhirter W, Green AC (1997) Risk factors for childhood melanoma in Queensland, Australia. Int J Cancer 70(1): 26-31
Yip BH, Pawitan Y, Czene K (2006) Parental age and risk of childhood cancers: a population-based cohort study from Sweden. Int J Epidemiol 35(6): $1495-1503$

Youl PH, Baade PD, Parekh S, English D, Elwood M, Aitken JF (2011) Association between melanoma thickness, clinical skin examination and socioeconomic status: results of a large population-based study. Int J Cancer 128(9): 2158-2165

This work is published under the standard license to publish agreement. After 12 months the work will become freely available and the license terms will switch to a Creative Commons Attribution-NonCommercial-Share Alike 3.0 Unported License. 